\title{
ON AN INEQUALITY OF NEHARI
}

A. M. FINK AND D. F. ST. MARY

Nehari [1, Theorem I] claims that if $[a, b]$ contains $n$ zeros of a nontrivial solution of $y^{(n)}+p_{n} y^{(n-1)}+\cdots+p_{1} y=0$, then

$$
\sum_{k=1}^{n} 2^{k}(b-a)^{n-k} \int_{a}^{b}\left|p_{k}\right|>2^{n+1} .
$$

In a private communication with one of the authors, Professor Nehari has indicated that the inequality is undecided since the argument given in [1] that Theorem II implies Theorem I is incorrect. It is the purpose of this note to show that (1) is correct for $n=2$. In fact, we prove a stronger result for

$$
y^{\prime \prime}+g y^{\prime}+f y=0 .
$$

THEOREM. Let $a$ and $b$ be successive zeros of a nontrivial solution to (2) where $f$ and $g$ are integrable. Then

$$
(b-a) \int_{a}^{b} f^{+}(x) d x-4 \exp \left(-\frac{1}{2} \int_{a}^{b}|g|(x) d x\right)>0
$$

and a fortiori

$$
(b-a) \int_{a}^{b} f^{+}(x) d x+2 \int_{a}^{b}|g|(x) d x>4 .
$$

If $a$ and $b$ are successive zeros, then there is a $c \in(a, b)$ such that $y^{\prime}(c)=0$. Nehari shows that

$$
1<(c-a) \int_{a}^{c}|f|+\int_{a}^{c}|g|,
$$

and a similar inequality for the interval $(c, b)$. The trick is to combine the two to get (4).

We start with an inequality which is stronger than (5). Consider the equation $\left(r y^{\prime}\right)^{\prime}+p y=0$ for $r>0$, with $r$ and $p$ integrable.

Lemma (SeE [2]). If $y(a)=0$ and $y^{\prime}(c)=0, a<c$, then

$$
1<\int_{a}^{c} r^{-1} \int_{a}^{c} p^{+}
$$

Received by the editors April 15, 1968. 
where $p^{+}(x)=\max \{p(x), 0\}$.

Proof. Let $|y(x)|=\max |y(t)|$. Then

$$
\begin{aligned}
(y(x))^{2} & \leqq\left(\int_{a}^{c}\left|y^{\prime}\right|\right)^{2}=\left(\int_{a}^{c} r^{-1 / 2}\left|\sqrt{r} y^{\prime}\right|\right)^{2} \\
& <\left(\int_{a}^{c} r^{-1}\right) \int_{a}^{c} r\left(y^{\prime}\right)^{2}=\left(\int_{a}^{c} r^{-1}\right) \int_{a}^{c} p y^{2} \\
& \leqq\left(\int_{a}^{c} r^{-1}\right) \int_{a}^{c} p^{+} y^{2} \leqq y^{2}(x) \int_{a}^{c} r^{-1} \int_{a}^{c} p^{+}
\end{aligned}
$$

from which the result follows. We have used the Schwarz inequality and the identity $\int_{a}^{c} r\left(y^{\prime}\right)^{2}=\int_{a}^{c} p y^{2}$ which may be verified by an integration by parts.

A similar result holds on $(c, b)$ where $y(b)=0$. Now we want to apply the lemma to the equation (2). Taking $r=\exp \int_{a}^{c} g$ and $p=r f$ we get

(6) $1<\int_{a}^{c} \exp \left(-\int_{a}^{t} g(s) d s\right) d t \int_{a}^{c} f^{+}(x) \exp \left(\int_{a}^{x} g(s) d s\right) d x$.

Writing this as a double integral we see that

$$
\begin{aligned}
1 & <\int_{a}^{c} \int_{a}^{c} \exp \left(\int_{t}^{x} g(s) d s\right) f^{+}(x) d x d t \\
& <\int_{a}^{c} \int_{a}^{c} \exp \left(\int_{a}^{c}|g|(s) d s\right) f^{+}(x) d x d t
\end{aligned}
$$

or

$$
1<(c-a) \int_{a}^{c} f^{+}(x) d x \exp \int_{a}^{c}|g|(s) d s .
$$

A similar result holds for the interval $(c, b)$ where $y(b)=0$.

In order to motivate a later inequality we observe that (7) implies (5) with $|f|$ replaced by $f^{+}$. Indeed, letting $A_{0}=\int_{a}^{c}|g|(x) d x$ and $A_{1}^{2}=(c-a) \int_{a}^{c} f^{+}(x) d x$, this claim is the statement that $A_{1}^{2}>\exp \left(-A_{0}\right)$ implies $A_{1}^{2}+A_{0}>1$. This follows from $e^{-x}+x \geqq 1$ for all $x \geqq 0$.

Now define $B_{1}^{2}=(b-c) \int_{c}^{b} f^{+}(x) d x$ and $B_{0}=\int_{c}^{b}|g|(x) d x$. Then we have $B_{1}^{2}>\exp \left(-B_{0}\right)$. Now the inequality that is related to (4) as (7) is to (5) is gotten from $4 \exp (-y / 2) \geqq-2 y+4, y \geqq 0$.

Proof of the Theorem. First we have that 


$$
\int_{0}^{b} f^{+}(x) d x=\frac{A_{1}^{2}}{c-a}+\frac{B_{1}^{2}}{b-c} \geqq \frac{\left(A_{1}+B_{1}\right)^{2}}{b-a}
$$

by elementary calculus. In fact, the right member is the minimum of the middle member as function of $c$. Thus the left-hand side of (3) is greater than

$$
\begin{aligned}
\left(A_{1}+B_{1}\right)^{2}-4 \exp [ & \left.-\frac{1}{2}\left(A_{0}+B_{0}\right)\right] \\
& \geqq A_{1}^{2}+B_{1}^{2}+2 A_{1} B_{1}-4 \exp \left[-\frac{1}{2}\left(A_{0}+B_{0}\right)\right] \\
& >\exp \left(-A_{0}\right)+\exp \left(-B_{0}\right)-2 \exp \left[-\frac{1}{2}\left(A_{0}+B_{0}\right)\right] \\
& =\left[\exp \left(-\frac{1}{2} A_{0}\right)-\exp \left(-\frac{1}{2} B_{0}\right)\right]^{2} \geqq 0 .
\end{aligned}
$$

This proves (3). Equation (4) follows from the inequality $4 \exp (-y / 2)$ $+2 y-4 \geqq 0$ for all $y \geqq 0$, where $y$ is replaced by $\int_{a}^{b}|g|$.

We remark that both inequalities (7) and (3) are more enlightening than their counterparts (5) and (4). In particular, they show that $x=(b-a) \int_{a}^{b} f^{+}$cannot be small unless $y=\int_{a}^{b}|g|$ is very large. In fact, as $x \rightarrow 0, y \rightarrow \infty$. This does not follow from (4). Finally, the inequality (3) is sharp since it reduces to Lyaponov's inequality when $g \equiv 0$, and this is known to be sharp, see [3].

AdDED IN PROOF. Professor P. Hartman has pointed out that (3) is announced in Levin, On linear second order differential equations, Soviet Math. Dokl. 4 (1963), 1814-1817. The content of this paper is an elementary proof of (3) and the observation that (4) follows from (3).

\section{REFERENCES}

1. Z. Nehari, "On an inequality of Lyapunov," in Studies in mathematical analysis and related topics, Stanford Univ. Press, Stanford, California, 1962, pp. 256-261.

2. D. F. St. Mary, Some oscillation and comparison theorems for $\left(r(t) y^{\prime}\right)^{\prime}+p(t) y=0$, J. Differential Equations (to appear)

3. W. Magnus and S. Winkler, Hill's equation, Interscience, New York, 1966.

Iowa State University 